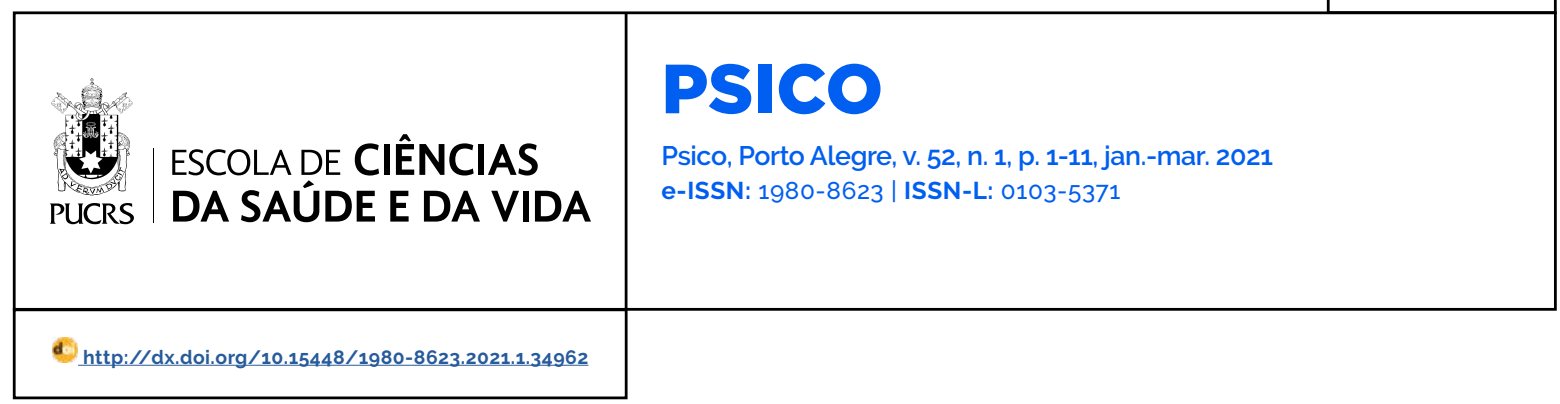

SEÇÃO: ARTIGO

\title{
Personalidade de insensibilidade e afetividade restrita em crianças vítimas de bullying
}

\author{
Callous-unemotional personality in children bullying victims \\ Personalidad de la insensibilidad y afectividad restringida en niños victimas de bullying
}

\section{Wandersonia Moreira \\ Brito Medeiros ${ }^{1}$ \\ orcid.org/0000-0002-2902-3957 \\ wandersoniamedeiros@hotmail.com}

Nelson Torro Alves ${ }^{1}$

orcid.org/0000-0003-3678-5762

nelsontorro@yahoo.com.br

\section{Carla Alexandra da}

Silva Moita Minervino ${ }^{1}$

orcid.org/0000-0002-3978-6958

carla_moita@hotmail.com

\section{Cyntia Diógenes}

\section{Ferreira $^{1}$}

orcid.org/0000-0001-8593-8177 cyntiadiogenes@gmail.com

\section{Bernardino Fernández}

Calvo 2

orcid.org/0000-0001-8080-5578 bfcalvo@usal.es

Recebido em: 23 jul. 2019. Aprovado em: 26 jun. 2020. Publicado em: 17 jun. 2021.

\section{(C) (i)}

Artigo está licenciado sob forma de uma licença Creative Commons Atribuição 4.0 Internacional.
Resumo: A personalidade com insensibilidade e afetividade restrita (Callous-Unemotional personality) é bem investigada nos agressores de bullying, porém pouco se sabe sobre essa característica nas vítimas. O presente estudo objetivou avaliar a personalidade de insensibilidade e afetividade restrita em crianças que vivenciaram situações de bullying. Participaram da pesquisa 60 crianças do $6 .{ }^{\circ}$ ano escolar divididas em grupos de agressores $(n=15)$, vitimas $(n=15)$, vitimas-agressores $(n=15)$, e grupo controle $(n=15)$. Foram aplicadas escalas de personalidade e de comportamento. Os resultados apontaram que as vitimas demonstraram um nivel mais baixo de personalidade com insensibilidade e afetividade restrita se comparado aos outros grupos, apresentando também maior nivel de comportamento pró-social. Torna-se, então, necessário investigar se níveis mais baixos deste traço de personalidade estão associados a maior passividade nas vítimas e, consequentemente, a uma maior probabilidade de serem alvo de bullying.

Palavras-chave: bullying, comportamento, empatia, falta de emoção

Abstract: The Callous-Unemotional personality it is well examined among bullying aggressors, however little is known regarding this characteristic in victims. The present study aimed to investigate the insensibility and coldness in children living under bullying circumstances. Sixty children of the $6^{\circ}$ school grade took part in the study, divided in groups of aggressors $(n=15)$, victims $(n=15)$, bully-victims $(n=15)$, and a control group $(n=15)$. Personality and behavior assessment scales were applied. Results revealed that victims showed a lower level of Callous-Unemotional personality compared to other groups, and also presented a higher level of pro-social behavior. It turns necessary to investigate if lower levels of those personality traits are associated to a higher passivity in victims and, consequently, to a greater probability of being target of bullying.

Keywords: bullying, behavior, emphatic, lack of emotion

Resumen: La personalidad con insensibilidad y afectividad restringida (Callous-Unemotional Personality) está bien investigada en agresores de bullying, pero poco se sabe sobre este rasgo en las víctimas. El presente estudio tuvo como objetivo evaluar la personalidad de insensibilidad y afectividad restringida en niños que experimentaron situaciones de bullying. Participaran de la investigación 60 niños del $6^{\circ}$ año escolar divididos en grupos de agresores $(n=15)$, víctimas $(n=$ $15)$, victimas-agresores $(n=15)$ y grupo controle $(n=15)$. Se aplicaron escalas de personalidad y conducta. Los resultados mostraron que las víctimas mostraron un menor nivel de personalidad con insensibilidad y afectividad restringida en comparación con los otros grupos, además de presentar un mayor nivel de conducta prosocial. Entonces se hace necesario investigar si los niveles más bajos de este rasgo de personalidad están asociados con una mayor pasividad en las victimas y, en consecuencia, a una mayor probabilidad de ser acosado. Palabras clave: bullying, conducta, empatía, falta de emoción 
O bullying é caracterizado pela presença de comportamento agressivo, que ocorre de forma intencional, repetitiva e persistente, e que resulta em prejuizos físicos ou psicológicos à vítima (DeCamp \& Newby, 2015; Olweus, 1994).

De acordo com a etiologia do comportamento agressivo, diferentes estratégias e metodologias de intervenção podem ser traçadas (Rigatti, 2016). A fim de compreender os fatores correlacionados ao bullying, torna-se essencial a avaliação dos aspectos comportamentais, psicopatológicos e de personalidade dos agressores e vítimas (Sourander et al, 2016). O bullying tem sido associado a problemas de comportamentos externalizantes, déficits na tomada de decisão, impulsividade e baixos niveis de comportamento pró-social (Evans-Lacko et al., 2017; Medeiros, et al., 2016; Navarro et al., 2016; Viding et al. , 2009).

Dentre os traços de personalidade estudados em crianças e adolescentes com transtorno de conduta ou personalidade antissocial estão a insensibilidade (callous) e a afetividade restrita (unemotional) (Assary et al., 2015; Frick et al., 2018; Rigatti, 2016). O constructo teórico da personalidade representando uma insensibilidade e afetividade restrita (callous-unemotional) inclui características como: falta de empatia, ausência de culpa e pobreza na expressão emocional, e foi criado para identificar precocemente traços de psicopatia em crianças e adolescentes (Kimonis et al., 2008; Ray \& Frick, 2018). A importância de tal constructo pode ser percebida através da inclusão do especificador "com emoção e comportamento pró-social limitados" para o diagnóstico de transtorno de conduta no DSM-5 (American Psychiatric Association [APA], 2014).

Jovens com traços de personalidade "callous-unemotional" aumentados tendem a utilizar a agressão como um método útil para estabelecer dominância, são mais propensos a focar nos resultados positivos de seu comportamento antissocial e a culpar os outros por seu mau comportamento (Frick et al., 2018). Por isso, têm-se investigado a influência destes traços de personalidade nos comportamentos de bullying.
Muñoz, et al. (2011) avaliaram 201 crianças de 11 e 12 anos através de medidas de bullying, empatia e por meio do Inventory of Callous-Unemotional Traits - ICU, que avalia traços de insensibilidade e afetividade restrita. Os resultados mostraram que crianças com maior pontuação na escala ICU apresentavam menores escores na medida de empatia e maior índice de envolvimento com bullying direto. No estudo de Ciucci e Baroncelli (2014), crianças com idade média de 11 anos e 8 meses demonstraram características de insensibilidade e afetividade restrita positivamente associada ao bullying, sendo essa associação mediada pela falta de empatia afetiva. Em alunos mais velhos (média de idade de 13 anos e 8 meses) a dimensão de insensibilidade da escala ICU estava diretamente relacionada ao bullying, sem associação com a medida de empatia.

Uma meta-análise conduzida por Mitsopoulou e Giovazolias (2015) investigou a empatia como sendo uma das caracteristicas de personalidade relacionadas ao comportamento de bullying. Embora sejam escassos os estudos que associaram a empatia e o bullying, os resultados indicaram que tanto a empatia cognitiva, quanto a afetiva, estavam negativamente relacionadas à perpetuação do bullying. De forma semelhante, Zych et al. (2019), através de uma revisão sistemática e de metanálise sobre empatia e traços de personalidade "callous-unemotional" relacionadas ao bullying escolar, encontraram que a perpetração de bullying foi correlacionada negativamente à empatia cognitiva e afetiva. Além disso, a perpetração também foi associada positivamente com traços de personalidade "callous-unemotional".

São consideradas vítimas as crianças que sofrem as agressões de maneira repetida. Alguns estudos acerca do padrão comportamental de crianças e de adolescentes vítimas de bullying indicam que essas, normalmente, apresentam características que podem contribuir para a inabilidade de reação diante das agressões, tal como àquelas relacionadas a um comportamento passivo, inibido, pouco sociável, inseguro, associado à baixa autoestima, ao medo e à vergonha (Lopes 
Neto, 2005; Miranda, 2012). Existe também um outro grupo, denominado de vítima/agressor, composto por crianças que, ao sofrerem o bullying, reagem de forma agressiva e passam também a praticar o comportamento de bullying com outras pessoas. Geralmente, são agressivas e provocativas, demonstrando maior probabilidade de apresentar sintomas depressivos, problemas de conduta, ansiedade, comportamentos externalizantes e impulsividade (Bandeira \& Hutz, 2010).

Crianças com baixa pontuação em traços de consciência e alta em Neuroticismo possuem mais probabilidade de vivenciarem afeto negativo durante o conflito entre pares, como maior irritação, atribuição de culpa ao agressor e perdoar menos. Essas reações estão relacionadas a niveis mais altos de vitimização (Bollmer et al., 2006).

Fontaine et al. (2018), em uma investigação longitudinal, avaliaram as associações entre os traços de personalidade "callous-unemotional" na infância (entre 7 e 12 anos) e a vitimização de pares na adolescência (14 anos). Os autores descobriram que os traços de personalidade "callous-unemotional" na infância prediziam a vitimização entre pares, mas que essa associação dependia das trajetórias dos traços e das formas de vitimização.

Em outro estudo, Wang et al. (2019) avaliaram as associações entre traços de personalidade com insensibilidade e afetividade restrita (callous-unemotional) e o envolvimento em bullying escolar em 613 adolescentes taiwaneses. Encontraram que niveis mais elevados dos traços de personalidade foram positivamente associados com maior risco de ser vitima de agressão física e roubo de pertences, ou de se tornar um agressor verbal, relacional e físico. Dessa maneira, destacam a importância da avaliação dos traços de personalidade "callous-unemotional" no desenvolvimento de programas para se detectar e prevenir o bullying escolar.

Os traços de personalidade de insensibilidade e de afetividade restrita são estudados comumente nos agressores, mas pouco investigados ainda em crianças que sofreram bullying. Entende-se que a compreensão do perfil de personalidade das vitimas pode contribuir para a criação de estratégias específicas para essa população, fornecendo subsídios para que elas se protejam ou modifiquem seu comportamento diante das agressões sofridas. Sendo assim, o presente estudo objetivou avaliar os traços de personalidade de insensibilidade e afetividade restrita em crianças que vivenciaram situações de bullying nos papéis de agressores, vítimas e vítimas-agressores.

\section{Método}

\section{Amostra}

Os critérios de inclusão estabelecidos foram: crianças com faixa etária entre 10 e 11 anos de idade, estudantes do $6 .^{\circ}$ ano do ensino fundamental II, matriculadas em escolas públicas e particulares de João Pessoa, PE, Brasil. Esta faixa etária foi selecionada pelos seguintes motivos: (a) maior incidência do comportamento de bullying neste periodo; (b) a Escala de Vitimização e Agressão entre Pares/EVAP, utilizada para mensurar o bullying, foi validada em crianças do ensino fundamental II; e (c) as crianças já entrariam em outra fase do desenvolvimento a partir de 12 anos de idade (adolescência). Foram definidos como critérios de exclusão: crianças com queixas de dificuldades visuais e/ou auditivas não corrigidas e portadores de déficit cognitivo comprometedor.

Inicialmente, a Escala de Vitimização e Agressão entre Pares (EVAP) foi aplicada em 225 alunos do $6 .^{\circ}$ ano de duas escolas públicas e uma escola particular. Após a exclusão das crianças que estavam na faixa etária da adolescência, foram entregues as escalas de preenchimento aos pais e o termo de consentimento livre-esclarecido. Trinta e nove crianças não participaram da análise, pois os pais não entregaram os questionários de Personalidade - ICU, nem o questionário de capacidades e dificuldades.

Também foram retiradas da amostra crianças que apresentavam pontuações limitrofes na escala EVAP e outliers. Participaram da análise final, 60 crianças (28 meninos) estudantes do 6. ano ensino fundamental, na faixa etária entre 10 e 11 anos de idade. A Tabela 1 apresenta a descrição da amostra. De acordo com a escala EVAP, os participantes foram divididos em quatro grupos: grupo agressores $(N=15)$, grupo vítimas $(N=15)$, grupo vitimas-agressores ( $\mathrm{N}=15)$ e grupo controle $(\mathrm{N}=15)$. 


\section{Instrumentos}

\section{Escala de Vitimização e Agressão entre Pares} (EVAP) (Cunha et al., 2009). A EVAP é uma escala de autorrelato, aplicável a estudantes da segunda fase do ensino fundamental, que investiga o processo de vitimização entre pares através de 18 itens. Os primeiros 10 itens mensuram comportamentos de agressão e os outros oito itens referem-se a comportamentos de vitimização no ambiente escolar. A criança deve selecionar a frequência (nunca = 1; quase nunca = 2; às vezes = 3; quase sempre 4; sempre $=5$ ) com que praticou ou sofreu determinado comportamento nos últimos seis meses. As respostas são somadas separadamente de acordo com as seguintes dimensões:

- agressão direta: itens 1, 2, 3, 4 e 6;

- agressão relacional: itens 7, 8, 9 e 10;

- agressão física indireta: item 5;

- vitimização: itens 11, 12, 13, 14, 15, 16, 17 e 18.

A presente pesquisa não investigou a dimensão "agressão física indireta", já que na escala tal dimensão é verificada através de um único item. Além das somas dos escores em cada dimensão de acordo com os valores sugeridos, foi realizada uma categorização dos participantes em subgrupos, de acordo com os escores em cada dimensão utilizando pontos de corte (Cunha et al., 2009). Os percentis maiores ou igual a 60 foram considerados como alta frequência do comportamento, os percentis entre 40 e 60 foram tidos como média frequência e os percentis abaixo de 40 foram considerados de baixa frequência. $\mathrm{Na}$ Tabela 1, são apresentados os pontos de corte.

Tabela 1 - Pontos de corte da Escala de Vitimização e Agressão entre Pares (EVAP)

\begin{tabular}{lcc}
\hline Dimensões & Percentil 40 & Percentil 60 \\
\hline Agressão direta & 7 & 9 \\
Agressão relacional & 6 & 8 \\
Vitimização & 12 & 16 \\
\hline
\end{tabular}

Pontuação com percentil $\geq 60$ : alta frequência do comportamento

Pontuação com percentil entre 40 e 60: média frequência Pontuação com percentil < 40: baixa frequência na dimensão
De acordo com os pontos de corte da Tabela 1, os grupos foram divididos da seguinte maneira:

- Grupo agressores ( $n=15)$ : crianças com alta frequência de comportamento de agressividade (escore $\geq 9$ nos itens de agressividade direta) e nivel baixo em vitimização (escore $\leq 12$ nos itens de vitimização) ou moderado (escore $\leq 16$ nos itens de vitimização foram incluídas neste grupo). As crianças que obtiveram altos escores somente nos itens de agressividade relacional, mas não apresentaram pontuações altas nos itens de agressividade direta, não foram incluidas nesse grupo. já que niveis moderados de avaliação relacional também foram encontrados no grupo controle.

- Grupo vítimas ( $n=15)$ : crianças com alta frequência de comportamento de vitimização (escore $\geq 16$ nos itens de vitimização) e baixo nivel de agressividade física (escore $\leq 7$ nos itens de agressividade direta) e relacional (escore $\leq 6$ nos itens de agressividade relacional).

- Grupo vítimas-agressores ( $n=15)$ : crianças com alta frequência de comportamento de agressividade (escore $\geq 9$ nos itens de agressividade direta) e de vitimização (escore $\geq 16$ nos itens de vitimização). Não foram incluidas neste grupo as crianças que pontuavam alto no indice de vitimização, mas que também apresentaram pontuações elevadas somente nos itens de agressividade relacional para que houvesse melhor caracterização do grupo e diferenciação do grupo de vítimas. Além disso, níveis moderados de avaliação relacional também foram encontrados no grupo controle.

- Grupo Controle (n=15): crianças que possuem baixa frequência de comportamento de agressividade (escore $\leq 7$ nos itens de agressividade direta) e de vitimização (escore $\leq 12$ nos itens de vitimização).

Inventory of Callous-Unemotional Traits (ICU) (Kimonis et al., 2008). A escala ICU foi preenchida pelos pais para verificar características de personalidade "callous-unemotional". A personalidade "callous-unemotional" refere-se a traços de personalidade "insensivel" e "incapacidade de sentir 
emoção" ou, também mencionado, "afetividade restrita" típica de individuos com alto índice de violência e antissociais

A ICU inclui 24 itens (por exemplo, "meu filho não mostra as emoções para os outros"), que são classificados em uma escala de Likert de quatro pontos de o (Absolutamente falso) a 3 (Absolutamente verdadeiro). A soma dos itens gera resultados de traços de indiferença, insensibilidade e afetividade restrita. Utilizou-se a soma dos escores brutos para comparação dos grupos.

Questionário de Capacidades e Dificuldades (Strengths and Difficulties Questionnaire-SDQ) (Goodman, 1997). O instrumento foi utilizado com o objetivo de analisar o perfil comportamental dos grupos. O Questionário de Capacidades e Dificuldades (Strengths and Difficulties Questionnaire-SDQ) é um questionário de rastreamento que visa avaliar a saúde mental de crianças e adolescentes (4 a 16 anos). Possui 25 itens, divididos em cinco subescalas: sintomas emocionais, problemas de conduta, hiperatividade, problemas de relacionamento com colegas e comportamento pró-social. Também é gerado um índice total de dificuldades que é a soma das subescalas (exceto sociabilidade). O instrumento pode ser utilizado em três versões: autopreenchimento, versão para pais e versão para professores. No presente trabalho foi utilizada a versão para pais. Cada item apresenta três alternativas de respostas, falso (zero ponto), mais ou menos verdadeiro (um ponto) e verdadeiro (dois pontos).

\section{Procedimentos}

Após a autorização da escola e dos responsáveis através dos termos de anuência e de consentimento livre esclarecido, a EVAP foi preenchida de forma coletiva em uma sala reservada disponibilizada pela escola. A partir dos resultados, as crianças foram selecionadas e os grupos foram definidos. O aplicador esteve presente durante todo preenchimento a fim de esclarecer dúvidas e evitar erros por falta de compreensão.

As escalas ICU e SDQ foram preenchidas pelos pais ou cuidadores. As escalas foram enviadas na mochila da criança, com autorização da escola, para os pais preencheram em casa e entregarem na coordenação da escola. Alguns enviaram também pela mochila das crianças. Neste caso, os avaliadores faziam contato telefônico para confirmar se realmente os pais ou cuidadores haviam recebido e preenchido.

A pesquisa foi aprovada pelo Comitê de Ética em Pesquisa do Hospital Universitário Lauro Wanderley da Universidade Federal da Paraíba (processo CAAE: 17883413.5.0000.5183). Todos os procedimentos éticos foram considerados de acordo com a Resolução 510/2016 do Conselho Nacional de Saúde. A participação foi voluntária e os participantes foram previamente informados que poderiam retirar seu consentimento a qualquer momento da pesquisa.

\section{Análise de dados}

Os dados foram submetidos ao teste de normalidade de Shapiro-Wilk para avaliar se apresentavam uma distribuição normal e ao teste de Levene para verificar a homogeneidade das variâncias. Como a maioria das variáveis não apresentou critérios para distribuição normal e algumas mantinham heterogeneidade das variâncias, optou-se por utilizar testes não paramétricos. Inicialmente, foram realizadas as estatísticas descritivas de frequência, porcentagem, medidas de tendência central e desvio-padrão.

Para comparação dos grupos (agressores, vítimas, vitimas-agressores, controle) utilizou-se o teste de Kruskal-Wallis. Quando foram encontrados resultados estatisticamente significativos $(p<0,05)$, foi aplicado o teste de Mann-Whitney para verificar as diferenças entre os grupos. Para controlar a probabilidade de erro tipo I, foi corrigido o valor crítico de alfa dividindo o nivel de significância $(0,05)$ pelo número de comparações. Portanto, considerou-se como estatisticamente significante apenas os valores de probabilidades inferiores a 0,0083 . Utilizou-se a equação $r=\frac{z}{\sqrt{n}}$, para converter o z-score em estimativa $r$ de tamanho do efeito (Fritz et al., 2013). Um tamanho do efeito de $r \leq 0.10$ foi considerado pequeno, $r \leq 0.30$ foi considerado médio e $r \geq 0.50$ foi considerado alto (Cohen, 1988). Todas as estatísticas descritivas e inferenciais foram calculadas usando o software IBM Statistical Package for the Social Sciences (SPSS) versão 21. 


\section{Resultados}

Com relação às caracteristicas sociodemográficas, um total de 28 (46,7\%) das 60 crianças que participaram da amostra eram do sexo masculino, com 34 (56,7\%) frequentadores de escolas privadas e 26 (43,3\%) frequentando escolas públicas em João Pessoa, PB, Brasil.
Uma análise estatistica preliminar não mostrou diferenças entre os gêneros em relação às variáveis investigadas no estudo ( $p>0.05$ ). A Tabela 2 mostra a pontuação média dos quatro grupos nas dimensões da escala EVAP. Na Tabela 3, são apresentados os dados dos quatro grupos de participantes nas escalas ICU e SDQ, assim como os resultados das análises estatísticas.

Tabela 2 - Média e desvio padrão de idade e escores nas dimensões da Escala de Vitimização e Agressão entre Pares (EVAP) nos quatro grupos

\begin{tabular}{lllll}
\hline \multicolumn{1}{c}{ Variável } & \multicolumn{1}{c}{ Agressor } & \multicolumn{1}{c}{ Vitima } & Vitima-Agressor & Controle \\
\hline & $M(D P)$ & $M(D P)$ & $M(D P)$ & $M(D P)$ \\
Idade & $10.73(0.45)$ & $10.87(0.35)$ & $10.80(0.41)$ & $10.73(0.45)$ \\
Agressão & $8.20(4.29)$ & $5.93(1.62)$ & $8.53(3.29)$ & $4.87(1.12)$ \\
Relacional & $11.73(2.01)$ & $6.47(0.91)$ & $12.67(1.71)$ & $6.40(1.18)$ \\
Agressão direta Vitimização & $13.33(2.19)$ & $20.73(4.90)$ & $23.73(5.78)$ & $11.07(2.05)$ \\
\hline
\end{tabular}

Nota: $M$ = média; $D P=$ desvio-padrão

Tabela 3 - Medidas de tendência central, variabilidade e Teste Kruskal-Wallis para o Questionário de Capacidades e Dificuldades (Strengths and Difficulties Questionnaire - SDQ) e Inventory of Callous-Unemotional Traits (ICU)

\begin{tabular}{|c|c|c|c|c|c|c|}
\hline \multicolumn{7}{|c|}{$\begin{array}{c}\text { Média (Desvio Padrão) } \\
\text { Intervalo de Confiança 95\% }\end{array}$} \\
\hline Variável & Agressor & Vítima & $\begin{array}{l}\text { Vitima- } \\
\text { agressor }\end{array}$ & Controle & $\begin{array}{l}\text { Kruskal- } \\
\text { Wallis }\end{array}$ & $\begin{array}{c}\text { Mann- } \\
\text { Whitney }^{*}\end{array}$ \\
\hline \multicolumn{7}{|l|}{ Escala SDQ } \\
\hline Sintomas emocionais & $3,14(1,74)$ & $5,80(3,07)$ & $2,79(2,25)$ & $4,00(2,77)$ & $p=0,02$ & $\begin{array}{l}p=0,0014 \\
r=-0,45(a) \\
p=0,008 \\
r=-0,48(b)\end{array}$ \\
\hline $\begin{array}{l}\text { Problemas de } \\
\text { comportamento }\end{array}$ & $3,07(2,65)$ & $1,87(1,80)$ & $2,73(1,43)$ & $2,27(1,62)$ & $p=0,47$ & \\
\hline Hiperatividade & $4,60(3,26)$ & $3.53(2,10)$ & $4,33(1,91)$ & $3.93(2,89)$ & $p=0,78$ & \\
\hline $\begin{array}{l}\text { Problemas de } \\
\text { relacionamento }\end{array}$ & $1,53(1,72)$ & $2,67(1,63)$ & $2,47(2,56)$ & $1,20(1,01)$ & $p=0,07$ & \\
\hline Comportamento pró-social & $7,07(2,40)$ & $9,27(0,96)$ & $7,87(1,59)$ & $8,33(1,54)$ & $p<0,01$ & $\begin{array}{l}p=0,001 \\
r=-0,59(a) \\
p=0,006 \\
r=-0,46(b) \\
p=0,047 \\
r=-0,31(c)\end{array}$ \\
\hline Total dificuldade & $12,80(8,24)$ & $14,07(7,16)$ & $12,80(6,71)$ & $11,40(5,27)$ & $p=0,78$ & \\
\hline
\end{tabular}




\begin{tabular}{|c|c|c|c|c|c|c|}
\hline \multicolumn{7}{|c|}{$\begin{array}{l}\text { Média (Desvio Padrão) } \\
\text { Intervalo de Confiança 95\% }\end{array}$} \\
\hline Variável & Agressor & Vítima & $\begin{array}{l}\text { Vitima- } \\
\text { agressor }\end{array}$ & Controle & $\begin{array}{l}\text { Kruskal- } \\
\text { Wallis }\end{array}$ & $\begin{array}{c}\text { Mann- } \\
\text { Whitney }^{*}\end{array}$ \\
\hline \multicolumn{7}{|l|}{ Escala ICU } \\
\hline Soma total & $18,38(8,03)$ & $13,07(4,87)$ & $22,13(8,19)$ & $20,47(8,71)$ & $p=0,01$ & $\begin{array}{l}p=0,043 \\
r=0,37(a) \\
p=0,002 \\
r=0,55(b) \\
p=0,015 \\
r=0,44(c)\end{array}$ \\
\hline Indiferença & $10,07(6,43)$ & $6,07(3,01)$ & $8,07(3,43)$ & $8,40(5,77)$ & $p=0,29$ & \\
\hline Insensibilidade & $4,33(5,37)$ & $3.53(4,05)$ & $6,47(5,86)$ & $4,47(3,18)$ & $p=0,25$ & \\
\hline Falta emoção & $6,07(2,18)$ & $3.73(3,01)$ & $6,00(3,31)$ & $5,013(2,87)$ & $p=0,14$ & \\
\hline
\end{tabular}

Nota: Comparação entre grupos: (a) Agressor x Vitima; (b) Vítima x Vitima/Agressor; (c) Vitima $\times$ Controle. *resultado com significância estatística $(p<0,0083$ ) ou tamanho do efeito médio ou grande $(r>30)$.

Escala SDQ = Questionário de Capacidades e Dificuldades (Strengths and Difficulties Questionnaire); Escala ICU = Inventory of Callous-Unemotional Traits.

\section{Inventory of Callous-Unemotional Traits (ICU)}

Os resultados do escore total da escala ICU indicam que o nivel de personalidade de insensibilidade e de afetividade restrita do grupo vitimas foi menor do que os demais grupos ( $x 2=$ 11.16, $p=0.01$ ). Ao realizar comparação entre os pares de grupos, percebe-se que as respostas do grupo vitimas se diferenciaram de maneira significativa do grupo vítimas-agressores $(U=$ $35.5, p=0.002, r=0.55)$. Quando se compara o resultado do grupo vítimas com os demais grupos (agressores e controle), apesar do valor de p não ter sido estatisticamente significativo, o tamanho do efeito foi moderado tanto na comparação com o grupo de agressores $(U=49.50, p=0.04, r=0.37)$, como com o grupo de crianças que não vivenciam situações de bullying $(U=49.50, p=0.01, r=0.44)$.

Não houve diferença entre os grupos de acordo com o preenchimento dos pais com relação aos subitens das escalas: traços de indiferença $(x 2=$ 3.71, $p=0.29)$, insensibilidade $(x 2=4.16, p=0.25)$ e falta de emoção $(x 2=5.43, p=0.14)$.

\section{Questionário de Capacidades e Dificuldades (SDQ)}

Na subescala de comportamento pró-social, diferenças entre os grupos foram encontradas ( $x 2$ $=11.35, p=0.01$ ) (Tabela 2). Análises entre pares de grupos mostraram que vitimas apresentaram maior escore do que o grupo de agressores $(U=$ 33.00, $p=0.001 r=-0.59)$ e vitima-agressores $(U=$ $54.5, p=0.006, r=-0.46)$. A comparação do comportamento pró-social entre vítimas e controle, apesar de não indicar significância estatística, demonstra um tamanho do efeito médio $(U=$ 73.5, $p=0.089, r=-0.31$.

Também houve diferença entre os grupos no item de questões emocionais ( $\chi 2=8.98, p=0.02$ ). O grupo de vitimas mais uma vez apresentou maior comprometimento que os demais, sendo estatisticamente significativa a diferença entre o grupo de vitimas-agressores $(U=45.00, p=0.008, r$ $=-0.48$ ). A comparação do item questões emocionais entre o grupo vitima com o grupo agressor, demonstrou tamanho do efeito médio $(r=-0.45)$. 
Não foram encontradas diferenças entre os grupos com relação ao escore total de dificuldades ( $\chi 2$ = 1.09, $p=0.780$ ), hiperatividade $(\chi 2=1.09, p=0.780$ ), problemas de comportamento $(\chi 2=2.52, p=0.472) \mathrm{e}$ problemas de relacionamento $(\chi 2=6.92, p=0.074)$.

\section{Discussão}

Nesse estudo, foram avaliados os niveis de personalidade com insensibilidade e afetividade restrita e problemas de comportamento em crianças que vivenciam situações de bullying. No presente estudo, as crianças do grupo agressores não demonstraram diferenças no índice de personalidade com insensibilidade e afetividade restrita se comparado às crianças do grupo controle.

Alguns autores apontam uma associação entre as caracteristicas de insensibilidade e de afetividade restrita e o comportamento de bullying. principalmente entre os adolescentes (Ciucci \& Baroncelli, 2014; Fontaine et al., 2018; Wang et al., 2019). Outras pesquisas na área destacam que a dimensão "indiferença" da escala ICU foi capaz de predizer tanto o bullying direto quanto o indireto (Muñoz et al., 2011; Viding et al., 2009).

Percebe-se, também, uma diferenciação entre o perfil de crianças com comportamentos disruptivos ou agressivos, de acordo com a presença ou não de traços de personalidade com insensibilidade. Crianças com Transtorno de Conduta que possuem traços de insensibilidade associados apresentaram resultados diferentes em avaliações cognitivas e afetivas se comparadas a crianças com o mesmo transtorno mas que não demonstravam sintomas de insensibilidade e de afetividade restrita, o que indica a existência de subtipos distintos dentro do mesmo transtorno (Anastassiou-Hadjicharalambous \& Warden, 2008). O tipo de agressão também se diferenciou a depender do nível de traços de personalidade com insensibilidade e afetividade restrita. No estudo realizado por Muñoz et al. (2008), os adolescentes com histórico de comportamento agressivo e que pontuavam alto na ICU revelaram um padrão de agressividade proativa, ou seja, premeditada e sem que houvesse necessariamente uma situação desencadeadora. Já os adolescentes que também apresentavam comportamento agressivo, mas pontuavam baixo na ICU, demonstravam, em sua maioria, um padrão de agressividade reativa. O segundo grupo era caracterizado como mais impulsivo, reagiam com agressividade de forma automática, defensiva e desproporcional, diante de alguma situação desencadeante, além disso, apresentaram maior associação com conflitos e desestrutura familiar.

Os resultados da soma total da ICU do grupo de vítimas, como esperado, demonstram um nivel mais baixo comparado ao grupo de agressores e vitima-agressores, porém ressalta-se o fato de as vitimas terem apresentado menor escore mesmo em relação ao grupo controle. Como os traços de insensibilidade e de afetividade restrita estão associados a um perfil de agressividade, comportamento antissocial, transtornos de conduta e outros transtornos disruptivos (Frick et al., 2018), poucos estudos têm sido realizado com as vítimas e com resultados incertos. Wang et al. (2019) encontraram uma relação positiva entre a vitimização e os traços de insensibilidade. Para explicar tais resultados, os autores trazem duas possiveis hipóteses: (1) ao analisarem a amostra, perceberam que muitos dos que sofriam intimidações, também intimidavam, reagindo às agressões sofridas; (2) sugeriram também que crianças vítimas de agressões físicas estariam tão adoecidas física e emocionalmente que teriam se tornado insensiveis ao sofrimento do outro.

Um outro estudo, também encontrou uma correlação positiva entre a vitimização e o item de indiferença da escala ICU, mas o fator vitimização também havia se correlacionado positivamente com o fator agressão, o que pode indicar grande quantidade de individuos considerados vítima-agressores na amostra (Ciucci \& Baroncelli, 2014). Zych et al. (2016) destacam a necessidade de concentrar esforços futuros de pesquisa no grupo de vítimas, já que a associação entre vitimização e traços de personalidade de insensibilidade e afetividade restrita foram investigadas em poucos estudos e, muitos deles, correlacionais, não diferenciando os grupos de vítimas e vitimas-agressores. Assim, ainda não é possivel compreender 
as repercussões sobre o comportamento quando os traços de personalidade de insensibilidade e afetividade restrita ocorrem em niveis mais reduzidos de intensidade.

No estudo realizado por Glasø et al. (2007), os autores alertaram sobre a importância de não negligenciar o fator personalidade na avaliação de vitimas de bullying, pois, apesar das características de personalidade não terem o poder de diferenciar vítimas de não vítimas, as crianças que sofreram bullying demonstraram mais sintomas emocionais e traços de introversão. As análises de características psicológicas, personalidade e vitimização em crianças de 10 a 13 anos mostraram que crianças com baixa pontuação no traço de Consciência e alta em Neuroticismo são mais propensas a experimentar efeitos negativos durante o conflito entre pares, como sentir-se mais irritado, culpar mais o agressor e perdoar menos, sendo tais reações relacionadas a niveis mais altos de vitimização (Bollmer et al., 2006). A percepção sobre a existência de um padrão de personalidade também havia sido descrito por Bernstein e Watson (1997). Segundo esses autores, as vitimas possuem características de personalidade e comportamento que os diferenciam dos pares, fazendo inclusive serem reconhecidos como vítimas em potencial.

No presente estudo, as vítimas apresentaram maior comportamento pró-social, relacionado a comportamentos de empatia (ajudar o outro, ser simpático, cuidar de crianças menores) e, também, apresentaram maiores niveis de problemas emocionais do que as crianças que praticam bullying. Os sintomas emocionais do questionário preenchido incluem características relacionadas a medos, preocupações, dores de cabeça, nervosismo e tristeza (Eastman et al., 2018). Resultados de estudos anteriores também mostram a relação entre vitimização e problemas emocionais, incluindo estresse pós-traumático e ideação suicida (Bannink et al., 2014; Zych et al., 2015; Ttofi, 2015).

Para Hising et al. (2019), o bullying afeta negativamente o estado emocional de todos os envolvidos, porém enquanto os grupos de agressores e de vítimas-agressores apresentaram maiores índices de transtornos de conduta, hiperatividade e impulsividade, o grupo de vitimas revelou niveis elevados de ansiedade e de depressão se comparado aos demais. Evidências de estudos longitudinais também indicam associações entre vitimização e sintomas somáticos como cefaleia, distúrbios do sono, dor de estômago, enurese, tontura, resfriado e dor, apoiando a hipótese de que o bullying ocasiona problemas de saúde e não o contrário (Lien et al., 2009).

Por fim, ressalta-se o padrão do grupo vítima-agressor. Apesar de sofrer bullying, essas crianças diferenciam-se daquelas que não reagem com agressividade (grupo vítimas), demonstrando menor pontuação na subescala de sintomas emocionais do questionário preenchido pelos pais, quando comparado às respostas referentes ao grupo vítimas. As crianças do grupo apresentaram também maior índice de personalidade com insensibilidade e afetividade restrita. Para Bandeira \& Hutz (2010), as crianças vítimas-agressoras possuem maior probabilidade de desenvolver comportamentos externalizantes e, diferente das crianças do grupo de agressores, não são populares, mas rejeitados pelos colegas.

\section{Considerações finais}

Diante das consequências negativas do comportamento de bullying na sociedade, é essencial compreender os fatores relacionados à agressão e à vitimização para se melhor estabelecer ações preventivas e remediativas. Este trabalho permitiu identificar diferenças relacionadas aos aspectos comportamentais e de personalidade com insensibilidade e afetividade restrita em crianças que estão envolvidas com o bullying. $O$ fato de as vitimas terem apresentado menor nivel de personalidade com insensibilidade e afetividade restrita gera a necessidade de se investigar se esse traço de personalidade está associado a maior passividade nas vítimas e, consequentemente, a uma maior probabilidade de serem alvo de bullying

Algumas limitações em nosso estudo precisam ser mencionadas, tais como o tamanho da amostra por grupo e o grupo restrito de uma série escolar de avaliação das crianças, o que neste 
caso não permite uma generalização do comportamento para crianças de outras faixas etárias. Outra limitação encontra-se no fato de algumas crianças do grupo agressor terem apresentado niveis moderados de vitimização, o que pode ter diminuido a apresentação do perfil de perpetrador de bullying nesse grupo. Nesses aspectos, recomenda-se que estudos futuros ampliem a avaliação da personalidade "callous-unemotional" em crianças de diferentes faixas etárias.

\section{Referências}

American Psychiatric Association. (2014). DSM-5: Manual diagnóstico e estatistico de transtornos mentais. Artmed Editora.

Anastassiou-Hadjicharalambous, X., \& Warden, D. (2008). Cognitive and affective perspective-taking in conduct-disordered children high and low on callous-unemotional traits. Child and Adolescent Psychiatry and Mental Health, 2(1), 16-27. https://doi.org/10.1186/1753-2000-2-16

Assary, E., Salekin, R. T., \& Barker, E. D. (2015). Big-Five and Callous-unemotional Traits in preschoolers. Journal of Psychopathology and Behavioral Assessment, 37(3), 371-379. https://doi.org/10.1007/s10862-014-9471-9

Bandeira, C. M., \& Hutz, C. S. (2010). As implicações do bullying na auto-estima de adolescentes. Psicologia Escolar e Educacional 14, 131-138. https://doi.org/10.1590/ $\underline{\mathrm{S} 1413-85572010000100014}$

Bannink, R., Broeren, S., van de Looij - Jansen, P. M., de Waart, F. G., \& Raat, H. (2014). Cyber and traditional bullying victimization as a risk factor for mental health problems and suicidal ideation in adolescents. PLOS ONE, 9(4), e94026. https://doi.org/10.1371/journal.pone.0094026

Bernstein, J. Y., \& Watson, M. W. (1997). Children who are targets of bullying: A victim pattern. Journal of Interpersonal Violence, 12(4), 483-498. https://doi. org/10.1177/088626097012004001

Bollmer, J. M., Harris, M. J., \& Milich, R. (2006). Reactions to bullying and peer victimization: Narratives, physiological arousal, and personality. Journal of Research in Personality, 40(5), 803-828. https://doi.org/10.1016/j. jrp.2005.09.003

Ciucci, E., \& Baroncelli, A. (2014). The emotional core of bullying: Further evidences of the role of callous-unemotional traits and empathy. Personality and Individual Differences, 67, 69-74. https://doi.org/10.1016/.jpaid.2013.09.033

Cunha, J. M., Weber, L. N. D., \& Steiner Neto, P. (2009). Escala de vitimização e agressão entre pares (EVAP). In Weber, L.\& Dessen, M. A. (Eds), Pesquiando em familia- instrumentos para coleta e análise de dados (pp. 108-120). Juá Editora.

DeCamp, W., \& Newby, B. (2015). From bullied to deviant: The victim-offender overlap among bullying victims. Youth Violence and Juvenile Justice, 13(1), 3-17. https:// doi.org/10.1177/1541204014521250
Eastman, M., Foshee, V., Ennett, S., Sotres-Alvarez, D., Reyes, H. L. M., Faris, R., \& North, K. (2018). Profiles of internalizing and externalizing symptoms associated with bullying victimization. Journal of Adolescence, 65, 101110. https://doi.org/10.1016/j.adolescence.2018.03.007

Evans-Lacko, S., Takizawa, R., Brimblecombe, N., King, D., Knapp, M., Maughan, B., \& Arseneault, L. (2017). Childhood bullying victimization is associated with use of mental health services over five decades: A longitudinal nationally representative cohort study. Psychological Medicine, 47(1), 127-135. https://doi.org/10.1017/So033291716001719

Frick, P. J., Robertson, E. L., \& Clark, J. E. (2018). Callousunemotional traits. In Martel, M. M., Developmental Pathways to Disruptive, Impulse-Control and Conduct Disorders (pp. 139-160). Elsevier. https://doi.org/10.1016/ B978-0-12-811323-3.00006-7

Fontaine, N. M. G., Hanscombe, K. B., Berg, M. T., McCrory, E. J., \& Viding, E. (2018). Trajectories of Callous-Unemotional Traits in Childhood Predict Different Forms of Peer Victimization in Adolescence. Journal of Clinical Child \& Adolescent Psychology, 47(3), 458-466. https:// doi.org/10.1080/15374416.2015.1105139

Glasø, L., Matthiesen, S. B., Nielsen, M. B., \& Einarsen, S. (2007). Do targets of workplace bullying portray a general victim personality profile? Scandinavian Journal of Psychology, 48(4), 313-319. https://doi.org/10.1111/j. 1467-9450.2007.00554.X

Goodman, R. (1997). The Strengths and Difficulties Questionnaire: A research note. Journal of Child Psychology and Psychiatry, 38(5), 581-586. https://doi. org/10.1111/j.1469-7610.1997.tb01545.x

Hysing, M., Askeland, K. G., La Greca, A. M., Solberg, M. E., Breivik, K., \& Sivertsen, B. (2019). Bullying involvement in adolescence: implications for sleep, mental health, and academic outcomes. Journal of interpersonal violence. https://doi:10.1177/0886260519853409

Kimonis, E. R., Frick, P. J., Skeem, J. L., Marsee, M. A., Cruise, K., Munoz, L. C., Aucoin, K. J.. Morris, A. S. (2008). Assessing Callous-Unemotional Traits in adolescent offenders: Validation of the Inventory of Callous-Unemotional Traits. International Journal of Law and Psychiatry, 31(3), 241-252. https://doi.org/10.1016/j.ijlp.2008.04.002

Lien, L., Green, K., Welander-Vatn, A., \& Bjertness, E. (2009). Mental and somatic health complaints associated with school bullying between 10th and 12th grade students; results from cross sectional studies in Oslo, Norway. Clinical Practice and Epidemiology in Mental Health, 5(1), 6-13. https://doi.org/10.1186/1745-0179-5-6

Medeiros, W., Torro-Alves, N., Malloy-Diniz, L. F., \& Minervino, C. M. (2016). Executive functions in children who experience bullying situations. Frontiers in Psychology, 7. 1197-1204. https://doi.org/10.3389/fpsyg.2016.01197

Miranda, R. de S. (2012). Bullying a partir de representações sociais de estudantes IDissertação de Mestrado, Universidade Federal da Paraibal. http://bdtd.biblioteca. ufpb.br/tde_busca/arquivo.php?codArquivo=2231

Mitsopoulou, E., \& Giovazolias, T. (2015). Personality traits, empathy and bullying behavior: A meta-analytic approach. Aggression and Violent Behavior, 21, 61-72. https://doi.org/10.1016/j.avb.2015.01.007 
Muñoz, L. C., Frick, P. J., Kimonis, E. R., \& Aucoin, K. J. (2008). Types of aggression, responsiveness to provocation, and callous-unemotional traits in detained adolescents. Journal of abnormal child psychology, 36(1), 15-28. https://doi.org/10.1007/s10802-007-9137-0

Muñoz, L. C., Qualter, P., \& Padgett, G. (2011). Empathy and bullying: Exploring the influence of callous-unemotional traits. Child Psychiatry \& Human Development, 42(2), 183- 196. https://doi.org/10.1007/s10578-010-0206-1

Navarro, R., Larrañaga, E., \& Yubero, S. (2016). Gender identity, gender-typed personality traits and school bullying: victims, bullies and bully-victims. Child Indicators Research, 9(1), 1-20. https://doi.org/10.1007/ s12187-015-9300-z

Olweus, D. (1994). Bullying at school. Springer US.

Ray, J. V., \& Frick, P. J. (2018). Assessing callous-unemotional traits using the total score from the inventory of callous-unemotional traits: A meta-analysis. Journal of Clinical Child \& Adolescent Psychology, 49(2), 190-199. https://doi.org/10.1080/15374416.2018.1504297

Rigatti, R. (2016). Adaptação transcultural e evidências de validação psicométricas do inventory of callous- unemotional traits (icu) para avaliação de traços de insensibilidade e afetividade restrita de adolescentes no brasil [Dissertação de mestrado não-publicada]. Universidade Federal do Rio Grande do Sul.

Sourander, A., Gyllenberg, D., Brunstein Klomek, A., Sillanmäki, L., Ilola, A.-M., \& Kumpulainen, K. (2016). Association of bullying behavior at 8 years of age and use of specialized services for psychiatric disorders by 29 years of age childhood bullying behavior and subsequent psychiatric disorderschildhood bullying behavior and subsequent psychiatric disorders. JAMA Psychiatry, 73(2), 159-165. https://doi.org/10.1001/ jamapsychiatry.2015.2419

Ttofi, M. M. (2015). Adolescent bullying linked to depression in early adulthood: Evidence supports early intervention. British Medical Journal, 350. https://doi. org/10.1136/bmj.h2694

Viding, E., McCrory, E. J., Blakemore, S.J., \& Frederickson, N. (2011). Behavioural problems and bullying at school: can cognitive neuroscience shed new light on an old problem? Update, 15(7), 289-291. https://doi. org/10.1016/j.tics.2011.05.001

Viding, E., Simmonds, E., Petrides, K. V., \& Frederickson, N. (2009). The contribution of callous-unemotional traits and conduct problems to bullying in early adolescence. Journal of Child Psychology and Psychiatry, 50(4), 471481. https://doi.org/10.1111/j.1469-7610.2008.02012.x

Zych, I., Ortega-Ruiz, R., Del Rey, R. (2015). Scientific research on bullying and cyberbullying: Where have we been and where are we going. Aggression and Violent Behavior, 24, 188-198. https://doi.org/10.1016/i. avb.2015.05.015

Zych, I., Ttofi, M. M., \& Farrington, D. P. (2016). Empathy and Callous-Unemotional Traits in different bullying roles: A systematic review and meta-analysis. Trauma, Violence, \& Abuse, 20(1), 3-21. https://doi. org/10.1177/1524838016683456
Wang, P.-W., Hsiao, R. C., Chen, L. M., Sung, Y.-H., Hu, H.-F., \& Yen, C.-F. (2019). Associations between callous-unemotional traits and various types of involvement in school bullying among adolescents in Taiwan. Journal of the Formosan Medical Association, 118(1, Part 1), 50-56. https://doi.org/10.1016/i.jfma.2018.01.003

\section{Wandersonia Moreira Brito Medeiros}

Mestre em Neurociência Cognitiva e Comportamento pela Universidade Federal da Paraíba (UFPB), em João Pessoa, PB, Brasil.

\section{Nelson Torro Alves}

Doutor em Psicobiologia pela Universidade de São Paulo (USP), em Ribeirão Preto, SP, Brasil; professor da Universidade Federal da Paraíba (UFPB), em João Pessoa, PB, Brasil.

\section{Carla Alexandra da Silva Moita Minervino}

Doutora em Ciências da Saúde pela Universidade Federal da Paraiba (UFPB), em João Pessoa, PB, Brasil; professora da Universidade Federal da Paraiba, em João Pessoa, PB, Brasil.

\section{Bernardino Fernández Calvo}

Doutor em Psicologia Clínica e da Saúde pela Universidade de Salamanca, e, Salamanca, Espanha; professor da Universidade Federal da Paraiba, em João Pessoa, PB, Brasil; professor da Universidad de Córdoba, em Córboda, Espanha.

\section{Cyntia Diógenes Ferreira}

Mestre em Neurociência Cognitiva e Comportamento pela Universidade Federal da Paraiba (UFP), em João Pessoa, PB, Brasil.

\section{Endereço para correspondência}

Wandersonia Moreira Brito Medeiros

Universidade Federal da Paraiba

Laboratório de Ciências Cognitivas e Percepção

Departamento de Psicologia

Av. Castelo Branco, s/n

Cidade Universitária, 58051900

João Pessoa, PB, Brasil

Os textos deste artigo foram revisados pela Poá Comunicação e submetidos para validação do(s) autor(es) antes da publicação. 